\title{
Comprensividad y prácticas de diferenciación educativa: creencias docentes y agrupamiento por niveles en Cataluña
}

\author{
Castejón,Alba \\ Departament de Pedagogia Sistemàtica i Social \\ Universitat Autònoma de Barcelona \\ Cerdanyola del Vallès, España \\ alba.castejon@uab.cat
}

\section{Resumen}

Esta comunicación tiene como objetivo comprender cómo el profesorado enmarca y concibe la educación comprensiva, particularmente las prácticas de agrupamiento del alumnado. La investigación se centra en la educación secundaria obligatoria en Cataluña donde coexiste una organización comprensiva de la educación obligatoria con estrategias informales de diferenciación educativa, como el agrupamiento por niveles. La ambigüedad de la normativa que regula el agrupamiento del alumnado, así como a la autonomía pedagógica que promueve la misma, posibilita la existencia de estrategias diferenciadoras a lo largo de la educación obligatoria, pues ampara a los centros educativos para que puedan agrupar a los y las jóvenes de la forma que consideren más adecuada. En este contexto, y con respecto a los procesos de toma de decisiones acerca del agrupamiento del alumnado, el profesorado es un actor clave cuyas creencias, discursos y prácticas condicionan las oportunidades educativas del alumnado. La investigación se basa en una etnografía desarrollada en dos institutos de educación secundaria con una alta complejidad social y que tienen modelos de agrupamiento distintos. Los resultados muestran que, aun situado en diferentes modelos de agrupamiento del alumnado, el profesorado de ambos institutos comparte creencias similares acerca de las prácticas de agrupamiento: en general, se critica al agrupamiento heterogéneo y se defiende el agrupamiento por niveles y la diferenciación, en base a la individualización, la meritocracia y la concepción de la educación secundaria como una etapa selectiva. Esta investigación demuestra el importante rol que las creencias docentes desarrollan en la implementación efectiva de las reformas comprensivas y orientadas a la equidad.

\section{Abstract}

This paper seeks to understand the ways in which teachers frame and conceive of comprehensive secondary education and, particularly, their beliefs regarding student grouping practices. The research is focused on lower secondary education in Catalonia (Spain), where students are not formally separated in tracks until the end of compulsory education. However, since the official normative calls for school autonomy in decisions regarding the organisation of students, the comprehensive model cohabits with informal mechanisms of student differentiation, such as ability grouping. Thus, teachers and other staff are important school actors in the processes of decision-making regarding student grouping and framing students' opportunities.

The research draws on an ethnographic approach and focuses on two socially disadvantaged secondary schools with different models of student grouping. Findings show that, although having different student grouping schemes, teachers in both schools share similar beliefs about student grouping practices: overall, they criticise mixed-ability grouping and advocate for ability grouping and differentiation. Teacher beliefs based on individualisation, meritocracy, and the view of lower secondary schooling as a selective stage, support their arguments for ability grouping. This research demonstrates the important role of teacher beliefs in effectively implementing comprehensive and equity-geared reforms.

Palabras clave: agrupamiento por niveles, creencias docentes, educación secundaria obligatoria, comprensividad.

Keywords: ability grouping, teacher beliefs, lower secondary education, comprehensiveness. 


\section{INTRODUCCIÓN}

La estructura de los sistemas educativos, en concreto el emplazamiento del alumnado en itinerarios educativos diferenciados, varía de manera importante entre los sistemas educativos. En los países europeos, existe un debate entre los sistemas comprensivos -aquellos donde los estudiantes están escolarizados bajo un tronco curricular común hasta el término de la educación obligatoria- y los sistemas diferenciados -aquellos donde los y las jóvenes son separados en itinerarios diferentes según las opciones postobligatorias (Green et al., 2001). Esta comunicación enmarca este debate en Cataluña, donde el sistema comprensivo está formalmente reconocido, pero donde existen prácticas de diferenciación educativa, como el agrupamiento por niveles.

En este contexto, la presente comunicación investiga por qué el profesorado aboga por agrupar a su alumnado por niveles, a pesar de la evidencia existente que demuestra que estas prácticas tienen efectos negativos en términos de eficacia y equidad, y cuestiona los planteamientos que, eludiendo las perspectivas críticas, asumen que las prácticas de agrupamiento del alumnado son cuestiones neutrales y técnicas.

\section{CONTEXTO Y ANTECEDENTES}

\subsection{El contexto catalán: el agrupamiento por niveles en un sistema comprensivo}

En el contexto catalán, estudiar el agrupamiento del alumnado en la educación secundaria es un aspecto clave ya que, a pesar de la naturaleza comprensiva del sistema, las prácticas de agrupamiento no están reguladas de forma explícita y, por lo tanto, dependen de decisiones que se toman a nivel escolar. Así, la ambigüedad de la normativa que regula el agrupamiento del alumnado, así como la autonomía pedagógica que se promueve en dicha normativa, ampara a los centros educativos para que puedan tomar las decisiones que más se adecuen a sus necesidades concretas y a las de sus estudiantes.

Este contexto favorece que existan múltiples y diversas prácticas de agrupamiento del alumnado, entre ellas el conocido como «agrupamiento por niveles», ampliamente utilizada en los institutos catalanes. Según datos de la última edición de PISA (OECD, 2015), en Cataluña un $26 \%$ del alumnado está escolarizado en centros que agrupan a sus estudiantes por niveles en todas las asignaturas (un $6 \%$ en el conjunto de España), porcentaje que sitúa a Cataluña como el territorio español donde más se utiliza -con diferencia- el agrupamiento por niveles.

\subsection{El agrupamiento por niveles en la investigación educativa}

El agrupamiento por niveles es la práctica de clasificar al alumnado, en el interior de los centros educativos, en diferentes grupos según su rendimiento académico o sus supuestas capacidades (Dupriez, 2010), bajo el argumento de que esta estrategia permite responder de forma adecuada a la diversidad académica de los y las jóvenes, orientada a proveer una enseñanza más efectiva para todo el alumnado. En este sentido, se arguye que el agrupamiento por niveles simplifica las condiciones de enseñanza-aprendizaje y permite al profesorado adaptar de forma más adecuada las actividades a las capacidades del alumnado, lo que se supone que conducirá, por sí mismo, a una mejora de sus aprendizajes. Sin embargo, la investigación educativa ha puesto de manifiesto en numerosas ocasiones que los efectos de esta estrategia -académicos, sociales, emocionales- no siempre son los deseados (Dupriez, 2010; Gamoran,2009).

En términos académicos, el agrupamiento por niveles no se ha correlacionado con una mejora del rendimiento medio del alumnado, pero sí con un aumento de las desigualdades académicas: esta práctica es especialmente nociva para aquellos chicos y chicas encuadrados en los grupos de «menor» nivel, e incrementa las diferencias de rendimiento entre el alumnado situado en los diferentes grupos (Duru-Bellat et al, 2004; Gamoran, 2009). En términos emocionales y actitudinales, la investigación revela que las experiencias y actitudes de los jóvenes hacia la escuela y la educación, difieren notablemente según el grupo en el que han sido encuadrados, siendo especialmente negativas entre aquellos chicos y chicas de los grupos con menor prestigio académico (Castejon, 2017; Hallam y Ireson, 2005; Oakes, 1985; Pàmies, 2013). 
Por último, la investigación también ha evidenciado que los procesos de clasificación del alumnado en los grupos de nivel conducen a una distribución desigual de los y las jóvenes según su perfil social y étnico. En este sentido, numerosos estudios demuestran que cuando se agrupa a los estudiantes "por capacidades» existe, también, una segregación social: aquellos y aquellas jóvenes de origen migrado o de clase trabajadora están sobrerrepresentados en los grupos de menor nivel (Boone y Van Houtte, 2013; Condron, 2007; Hallinan, 1994; Oakes, 1985). Esta distribución desigual, tal y como han mostrado estos estudios, se debe, en parte, a la activación, en estos procesos de clasificación, de unas expectativas docentes sesgadas social y culturalmente. En definitiva, la investigación educativa ha asociado de forma reiterada el agrupamiento por niveles con las desigualdades educativas, no solamente con su reproducción, sino como mecanismo para su amplificación.

\section{METODOLOGÍA}

Los datos que se presentan en esta comunicación proceden de una etnografía escolar llevada a cabo en dos institutos de educación secundaria de una gran ciudad de la Región Metropolitana de Barcelona. La selección de los centros estuvo basada en dos criterios. El primer criterio fue el tipo de agrupamiento del alumnado. Teniendo en cuenta que la tipología de agrupamiento del alumnado se articula en un continuum de posibilidades, se escogieron dos «casos extremos» (Goetz y LeCompte, 1998) para desarrollar una comparación: uno de los centros agrupa a sus estudiantes de forma heterogénea mientras que el otro aplica el agrupamiento por niveles para todas las asignaturas y en toda la ESO.

El segundo criterio utilizado para la selección de los centros fue su composición social, estimada a partir del porcentaje de alumnado en situaciones socioeconómicas desfavorecidas, el porcentaje de alumnado de nacionalidad no española y el porcentaje de alumnado que recibe ayudas para libros o material escolar. A diferencia del primer criterio de selección, este segundo criterio se definió con la voluntad de seleccionar dos casos similares. Se seleccionaron dos institutos con una composición social mayormente representada por jóvenes de clase trabajadora y de origen inmigrante.

El trabajo de campo se inició en octubre de 2014 y se prolongó hasta finales de julio de 2015, con una estada periódica en los centros (dos días a la semana), principalmente durante el horario lectivo. Si bien la principal técnica de recogida de información fue la observación participante en una amplia diversidad de espacios y tiempos escolares (aulas, recreos, reuniones docentes, etc.), también se llevaron a cabo entrevistas semiestructuradas, cuestionarios, y análisis documental. Concretamente, se llevaron a cabo 55 entrevistas semiestructuradas con diferentes actores (estudiantes, profesorado, directores, coordinadores académicos, inspección educativa y personal de apoyo educativo).

\section{RESULTADOS: CREENCIAS DEL PROFESORADO ACERCA DE LAS PRÁCTICAS DE AGRUPAMIENTO}

El objetivo principal de esta comunicación es identificar las razones por las cuales el profesorado, a pesar de la numerosa evidencia de los efectos contraproducentes del agrupamiento por niveles, defiende esta estrategia. En concreto, se pretende responder la siguiente cuestión: si la investigación educativa ha mostrado que el agrupamiento por niveles tiende a (re) producir las desigualdades en los resultados académicos, las experiencias escolares y las oportunidades, ¿por qué hay docentes que abogan por esta práctica?

En este apartado se examinan las creencias docentes acerca de las prácticas de agrupamiento del alumnado, explorando los siguientes aspectos: las concepciones sobre «la diversidad» del alumnado; las implicaciones de la diferenciación curricular durante la educación obligatoria; las percepciones sobre la eficacia y la eficiencia del agrupamiento heterogéneo y por niveles; y las valoraciones, en términos de justicia educativa, de las prácticas de agrupamiento del alumnado.

\subsection{El «problema de la diversidad»}

El trabajo de campo muestra que el profesorado concibe la diversidad del alumnado desde una perspectiva normativa que refleja una concepción esencialista de las capacidades y de las diferencias. 
De forma mayoritaria, el profesorado tiende a referirse a parámetros de «normalidad» para clasificar a los estudiantes entre aquellos que "pueden seguir el currículum ordinario» y aquellos de quien se asume que no tienen suficientes habilidades para seguirlo. Más allá de esta distinción dicotómica y simplificada, la diversidad es también vista como un fenómeno problemático dado que implica una presencia importante de estudiantes que distan notablemente de las características deseadas del «alumno ideal» (Becker, 1952). Estas concepciones de la diversidad implican disposiciones concretas sobre cómo ésta debe ser gestionada en la escuela y las aulas. Esta concepción restringida y problemática de la diversidad, así como los elementos que se desarrollan a continuación, explican que el profesorado muestre sus preferencias para el agrupamiento por niveles, frente a la ineficacia e injusticia percibidas de las prácticas de agrupamiento heterogéneo.

\subsection{Fuertes creencias sobre la necesidad de diferenciación}

A lo largo del trabajo de campo, se identificó entre los discursos docentes una concepción de la educación obligatoria que se aleja de los principios de la comprensividad. De hecho, la reforma comprensiva desarrollada en España a partir de la LOGSE se considera, no solamente inefectiva, sino incoherente e incluso absurda. En este sentido, el profesorado de ambas escuelas considera que los contenidos de la educación obligatoria deberían ser diferentes según las perspectivas educativas futuras de los estudiantes, cuestionando la necesidad y la conveniencia de un currículo común que proporcione a todos los estudiantes las mismas habilidades mínimas y que los sitúe en una posición de igualdad relativa al final de la educación obligatoria.

\subsection{La percepción de ineficacia del agrupamiento heterogéneo}

En relación a sus responsabilidades profesionales, el profesorado afirma que su rol consiste en promover actividades de enseñanza-aprendizaje que den respuesta a las necesidades individuales de sus estudiantes. Sin embargo, dada su percepción de la diversidad como un constructo dicotómico y problemático, y la creencia arraigada de la necesidad de diferenciación, el profesorado considera que la diversidad, y por tanto el agrupamiento heterogéneo, agrega «demasiada complejidad» en el trabajo docente y frustra sus posibilidades de desarrollar su trabajo de forma adecuada. Si bien parte del profesorado argumenta que esta complejidad se debe a la falta de herramientas y recursos para enseñar en aulas social y académicamente diversas, en general, el agrupamiento por niveles se concibe como una forma de reducir la diversidad «indeseable» y de hacer más efectiva la tarea docente.

\subsection{La percepción de injusticia del agrupamiento heterogéneo}

Más allá de las percepciones de ineficacia del agrupamiento heterogéneo, esta estrategia también se cuestiona en términos de justicia. El profesorado de ambas escuelas argumenta que el agrupamiento heterogéneo es injusto para aquellos y aquellas jóvenes que «quieren estudiar» porque perjudica sus procesos de aprendizaje. En ambos institutos, el profesorado arguye un discurso meritocrático para argumentar que agrupamiento por niveles permite a las escuelas «salvar a los estudiantes que se pueden salvar» y recompensar a quienes «merecen» el éxito, mientras que el agrupamiento heterogéneo socava su «derecho a estudiar, a aprender y a tener éxito». Sin embargo, no se presta mucha atención a aquellos estudiantes para los que el agrupamiento por niveles implica una pérdida de referentes y experiencias positivas.

\section{DISCUSIÓN Y CONCLUSIONES}

Esta comunicación está orientada a identificar las creencias de los docentes con respecto a las decisiones de agrupamiento que surgen en dos institutos donde se desarrollan diferentes modelos de agrupamiento del alumnado. El estudio muestra que, a pesar de trabajar en diferentes modelos de agrupamiento, el profesorado de ambos centros comparten creencias similares sobre las prácticas de agrupamiento: en general, critican el agrupamiento heterogéneo -uno de los principios de 
la comprensividad- y abogan por el agrupamiento y la diferenciación por capacidades. El estudio también identifica que los argumentos que usa el profesorado para defender el agrupamiento por niveles -y para cuestionar el agrupamiento heterogéneo- se basan en una concepción restringida, dicotómica y problemática de la diversidad y en creencias sólidas sobre la meritocracia y la selección, que están profundamente arraigadas en la mentalidad del profesorado y que desafían directamente al principios de comprensividad.

Concretamente, el profesorado tiende a construir una dicotomía entre los y las jóvenes "capaces» y «motivados», los «merecedores», y los y las jóvenes «no aptos» y "desmotivados», los «no merecedores», para cada uno de los cuales la educación debe proporcionar respuestas diferenciadas. El agrupamiento por niveles emerge como una estrategia "adecuada» para que los docentes se adapten a las necesidades y los méritos individuales de sus estudiantes: les permite diferenciar currículum de acuerdo con las capacidades, motivaciones y expectativas futuras de los estudiantes, y construye un sistema de «recompensa-castigo», que encaja dentro del ideal de justicia meritocrática que tiene el profesorado (Clyq et al., 2014; Tarabini, 2015).

La investigación actual también ha demostrado que las decisiones con respecto a las prácticas de agrupamiento del alumnado no son cuestiones técnicas ni neutrales, sino que se deben discutir desde un plano político. Como otros autores han señalado previamente (Oakes, 1994; Oakes et al., 1997), el agrupamiento por niveles no puede tratarse simplemente como una estrategia organizativa o como una decisión técnica. El agrupamiento por niveles es, por el contrario, una cuestión política que genera privilegios y desventajas por sí misma y refleja la idea de jerarquía y meritocracia como un «proceso de clasificación natural» (Oakes et al., 1997). Estos hechos se evidencian a través de los argumentos que el profesorado expone con respecto a sus preferencias para el agrupamiento por niveles.

En conclusión, esta investigación demuestra que, en las reformas educativas, es esencial tener en cuenta la necesidad de cambios en múltiples niveles. En el caso de la reforma comprensiva en el contexto español, el cambio en la estructura del sistema educativo ha demostrado ser insuficiente para alcanzar los objetivos de la comprensividad, es decir, lograr la igualdad de oportunidades educativas. Los mecanismos informales de selección temprana, apoyados activamente por parte de los docentes, aún prevalecen en los centros educativos. Esta investigación muestra, así, el importante papel de las creencias de los docentes en la implementación efectiva de reformas integrales y orientadas a la equidad.

Por lo tanto, es esencial centrarse en los cambios en las creencias del profesorado para abordar las desigualdades que se (re)producen dentro de las escuelas, abordar cambios en la formación docente inicial y promover programas para proporcionar al profesorado herramientas analíticas para comprender su papel en la promoción o reducción de las desigualdades educativas.

Asimismo, hay que tener en cuenta que la implementación de la comprensividad necesita una reconceptualización del currículo, una distribución más justa de los recursos y una mayor comprensión de las dificultades que enfrentan el profesorado y las escuelas para proporcionar educación de calidad para todo el alumnado en grupos heterogéneos.

\section{REFERENCIAS BIBLIOGRÁFICAS}

Becker, H. S. (1952). Social-Class Variations in the Teacher-Pupil Relationship. Journal of Educational Sociology, 25(8), 451-465. (X)

Boone, S., y Van Houtte, M. (2013). Why are teacher recommendations at the transition from primary to secondary education socially biased? A mixed-methods research. British Journal of Sociology of Education, 34(1), 20-38.

Castejon, A. (2017) Expectativas docentes, agrupamiento del alumnado y segregación escolar. Una etnografía en entornos de alta complejidad social en Cataluña. Tesis Doctoral. Departamento de Pedagogía Sistemática y Social, Universidad Autónoma de Barcelona. 
Clycq, N., Nouwen, W. M. A., \& Vandenbroucke, A. (2014). Meritocracy, deficit thinking and the invisibility of the system: Discourses on educational success and failure. British Educational Research Journal, 40(5), 796-819.

Condron, D. J. (2007). Stratification and Educational Sorting: Explaining Ascriptive Inequalities in Early Childhood Reading Group Placement. Social Problems, 54(1), 139-160.

Dupriez, V. (2010). Methods of Grouping Learners at School. Paris: UNESCO.

Duru-Bellat, M., Mons, N., \& Suchaut, B. (2004). Caractéristiques des systèmes éducatifs et compétences des jeunes de 15 ans. L'éclairage des comparaisons entre pays (Cahier de l'IREDU No. Les Cahiers de l'IREDU, No.66).

Gamoran, A. (2009). Tracking and Inequality: New Directions for Research and Practice (No. 2009-6, WCER Working Paper). Madison: University of Wisconsin-Madison, Wisconsin Center for Education Research.

Goetz, J. P., \& LeCompte, M. D. (1998). Etnografía y diseño cualitativo en investigación educativa. Madrid: Morata.

Green, A., Leney, T., y Wolf, A. (2001). Convergencias y divergencias en los sistemas europeos de educación y formación profesional. Barcelona: Ed. Palomares.

Hallam, S., \& Ireson, J. (2005). Secondary School Teachers' pedagogic practices when teaching mixed and structured ability classes. Research Papers in Education, 20(1), 3-24.

Hallinan, M. T. (1994). Tracking: From Theory to Practice. Sociology of Education, 67(2), 79-91.

Oakes, J. (1985). Keeping Track: how schools structure inequality. New Haven: Yale University Press.

Oakes, J. (1994). More Than Misapplied Technology: A Normative and Political Response to Hallinan on Tracking. Sociology of Education, 67(2), 84-91.

Oakes, J., Wells, A. S., Jones, M., \& Datnow, A. (1997). Detracking: The Social Construction of Ability, Cultural Politics, and Resistance to Reform. Teachers College Record, 98(3), 482- 510.

Pàmies, J. (2013). El impacto de los agrupamientos escolares. Los espacios de aprendizaje y sociabilidad de los jóvenes de origen marroquí en Barcelona. Revista de Educación, 362, 133-158.

Tarabini, A. (2015). La meritocracia en la mente del profesorado: un análisis de los discursos docentes en relación al éxito, fracaso y abandono escolar. Revista de La Asociación de Sociología de La Educación, 8(3), 349-360. (X) 